\title{
Compartmentalized Ras signaling in lymphocytes
} MR Philips

Address: NYU Scool of Medicine, Departments of Medicine, Cell Biology and Pharmacology, New York, USA

from 12th Joint Meeting of the Signal Transduction Society (STS). Signal Transduction: Receptors, Mediators and Genes

Weimar, Germany. 29-31 October 2008

Published: 26 February 2009

Cell Communication and Signaling 2009, 7(SuppI I):A83 doi:10.1 186/1478-8IIX-7-SI-A83

This abstract is available from: http://www.biosignaling.com/content/7/SI/A83

(c) 2009 Philips; licensee BioMed Central Ltd.

Ras proteins are peripheral membrane proteins targeted via post-translational modification of a C-terminal CAAX sequence. Although the plasma membrane (PM) is one organelle to which Ras proteins are targeted, this compartment is no longer considered the only platform from which Ras can signal. The palmitoylated isoforms, Nras and Hras, cycle between the Golgi apparatus and PM as a consequence of a palmitoylation/depalmitoylation cycle. We have studied dual compartment signaling in T lymphocytes. Ras activation as a consequence of antigen receptor (TCR) engagement on T lymphocytes is required for T-cell development, selection and function. Lymphocyte function-associated antigen-1 (LFA-1) mediates lymphocyte adhesion, stabilization of the immune synapse and bidirectional signaling. Using a fluorescent biosensor we found that TCR activation with or without costimulation of CD28 led to activation of Ras only on the Golgi apparatus, whereas co-stimulation with LFA-1 induced Ras activation on both the Golgi and the PM. Ras activation on both compartments required RasGRP1, an exchange factor regulated by calcium and diacylglycerol (DAG), but phospholipase C activity was required only for activation on the Golgi. Engagement of LFA-1 increased DAG levels at the PM by stimulating phospholipase D (PLD). PLD2 and phosphatidic acid phosphatase were required for Ras activation on the PM. Thus, LFA-1 acts through PLD2 to reshape the pattern of Ras activation downstream of the TCR. 